\title{
Analysis of Strain Localization during Tensile Tests by Digital Image Correlation
}

\section{by B. Wattrisse, A. Chrysochoos, J.-M. Muracciole and M. Némoz-Gaillard}

\begin{abstract}
This paper presents an imaging technique developed to study the strain localization phenomena that occur during the tension of thin, flat steel samples. The data are processed using digital speckle image correlation to derive the two in-plane components of the displacement vectors. The authors observe that the calculation of the intercorrelation function reveals a systematic error and propose a numerical method to limit its influence. Plastic incompressibility and thin-sheet assumptions are used to derive the third displacement component and, hence, the various strain and strain rate components. Numerous checks are presented at each step in processing the data to determine the final accuracy of the strain measurements. It is estimated that this accuracy is quite sufficient to track the inception and the development of localization. Examples of possible application are presented for mild steels whose strain localization mechanisms appear to be precocious and gradual.
\end{abstract}

KEYWORDS—Digital image correlation, strain rate measurement, necking, material behavior, steel

Over the past two decades, the simulation tools used in mechanics of materials have become increasingly powerful. Progress made in the field of scientific computation is giving rise to a new branch of mechanics called computational mechanics of solids. ${ }^{1}$ It allows the use of more realistic, and also more complex, models of behavior.

Of course, the consistency of the simulation results depends very much on the validity of the constitutive equations implemented in the computational codes. These phenomenological equations are, most often, identified on the basis of experiments carried out on particular structures for which stress and strain patterns are supposed to be known. For instance, we assume that strain and stress fields are homogeneous in the gage part of a sample during a simple tensile test. This homogeneity hypothesis, often implicitly assumed, is necessary to estimate the stress and the strain from load and displacement measurements. Naturally, as soon as a strain localization occurs-local necking, Lüders bands, transformation bands and so on-the mechanical fields are

\footnotetext{
B. Wattrisse is an Assistant Professor, A. Chrysochoos is a Professor and J.-M. Muracciole is an Assistant Professor, Laboratoire de Mechanique et Genie Civil, Universite Montpellier 2, 34095 Montpellier, Cedex 05, France. M. Némoz-Gaillard is an Assistant Professor, Laboratoire de Mechanique et de Metrologie, Ecoles des Mines d'Ales, 30319 Ales, France.
}

no longer homogeneous. These localization phenomena have long been under investigation by many authors from experimental, theoretical and numerical points of view. ${ }^{2-10}$ These works call for the detailed knowledge of the constitutive equations. Consequently, it is of a great importance to be able to detect, with even greater precision, the onset of such localization mechanisms.

The computational resources currently available offer new and powerful possibilities of experimental data processing. For instance, optical methods using digital image correlation provide whole-field displacement data for plane structures. ${ }^{11-14}$ The most precise methods take into account the deformation of the surface due to straining between two images. ${ }^{13}$ Nevertheless, these methods use iterative algorithms and generally require considerable computation time. In addition, to track the localization development precisely, the strain field must be determined on high-resolution grids. This fact led us to develop a suitable direct correlation method. It enables such resolutions to be achieved within a reasonable computation time using a standard microcomputer. The third displacement component can be obtained by assuming material incompressibility and depthwise homogeneity of the strain field (thin-sheet assumption). The strain and strain rate tensors are derived by differentiation. Because the displacement fields are discrete and noisy, a local polynomial fitting method is implemented to estimate the space and time derivatives.

This paper is divided into six sections, followed by concluding comments. The first three focus on the different steps in the image processing. The first is devoted to computation of the two in-plane components of the displacement vector. The second describes the calculation of the out-of-plane component. The third presents the differentiation method. The fourth section deals with the tests developed to check the accuracy of the displacement and strain measurements. Next, the experimental setup is introduced. Finally, potential applications of the experimental approach are proposed through results describing the localization development in tensile tests performed on standard thin, flat steel samples.

\section{In-plane Components of the Displacement Vector}

\section{Grid Step Definition}

The recorded speckled images correspond to noisy twodimensional discrete signals defined by the distribution of light intensity on the sample surface. The displacement field is computed at each point $M\left(i_{0}, j_{0}\right)$ of a virtual grid defined in the reference configuration. To achieve high spatial 
resolution, the grid step of the mesh (GS) is generally chosen between 2 and 5 pixels corresponding to about $10^{4}$ points of measurements per couple of images.

\section{Intercorrelation Function $\bar{\varphi}$}

To determine the displacement at each point of the grid, we choose to perform a direct correlation computation and use a normalized intercorrelation function $\bar{\varphi}$. Between two images and $I_{1}$ and $I_{2}$, separated by a small strain increment, $\bar{\varphi}$ is written as

$$
\begin{aligned}
& \bar{\psi}(k, l) \\
& k, l \in[-R Z, R Z]= \\
& \sum_{i, j=-C Z / 2}^{C Z / 2} I_{1}\left(i_{0}+i, j_{0}+j\right) \cdot I_{2}\left(i_{0}+i+k, j_{0}+j+l\right) \\
& \sqrt{\sum_{i, j=-C Z / 2}^{C Z / 2} I_{1}\left(i_{0}+i, j_{0}+j\right)^{2}} \\
& \frac{1}{\sqrt{\sum_{i, j=-C Z / 2}^{C Z / 2} I_{2}\left(i_{0}+i+k, j_{0}+j+l\right)^{2}}}
\end{aligned}
$$

where $C Z$ is the correlation zone (i.e., $M$ 's neighborhood corresponding to a local optical signature of grid point $M$ ) and $R Z$ is the research zone (i.e., $M$ 's neighborhood where the optical signature is tracked). These zones correspond to the domains of variation of $(i, j)$ and $(k, l)$, respectively.

The intercorrelation function $\bar{\varphi}$ is computed for multiple pixel shifts $(k, l)$. So the displacements are estimated with a 1-pixel resolution. To achieve higher resolutions, the discrete intercorrelation function is interpolated in the neighborhood of its discrete maximum by a quadratic polynomial. ${ }^{16}$

\section{Choice of $\mathrm{CZ}$ and $\mathrm{RZ}$}

The computation time for the intercorrelation function is related to $C Z$ and $R Z$ : it increases approximately as the square power of these two quantities.

The parameter $C Z$ affects the accuracy of the displacement measurement; the use of larger $C Z$ tends to increase the accuracy. A compromise has to be found between speed and accuracy. For this study, we choose a $C Z$ of approximately $20 \times 20$ pixels.

To minimize the computation time, small values of $R Z$ should also be used. But, in the case of large transformations, the incremental displacement may become too great. To maintain a short CPU time, we first compute a rough estimate of the displacement field on a coarse grid ( $G S=25$ to 50 pixels) using a sufficiently large $R Z$. Then, we compute the displacement on a finer grid using an interpolation of the previously computed data as a first estimate of the displacement field.

For example, between two $1024 \times 1024$ images homogeneously deformed (tensile strain state, longitudinal strain: 10 percent), $R Z$ should be at least 103 pixels. For a displace- ment field spanning $n=10^{4}$ points, the computation time is around $22.10^{3} \mathrm{~s}$ for the complete calculation, whereas it is only around $222+42=264 \mathrm{~s}$ when an interpolation of the displacement field calculated on $n=10^{2}$ points is used to compute the whole displacement field (see Table 1). Using the first estimate of the displacement field, $R Z$ ranges from 3 to 5 pixels.

\section{Out-of-plane Components of the Displacement Vector}

Assuming the plastic incompressibility, the out-of-plane component of the displacement vector can be deduced from the in-plane components. In the finite transformations framework, the local equation of the volume balance leads to

$$
\operatorname{det}(\mathbf{F}(M))=1,
$$

where $\mathbf{F}(M)$ is the deformation gradient tensor at point $M$. Its representative matrix is defined by

$$
F=\left(\begin{array}{ccc}
1+\frac{\partial u}{\partial x} & \frac{\partial u}{\partial y} & \frac{\partial u}{\partial z} \\
\frac{\partial v}{\partial x} & 1+\frac{\partial v}{\partial y} & \frac{\partial v}{\partial z} \\
\frac{\partial w}{\partial x} & \frac{\partial w}{\partial y} & 1+\frac{\partial w}{\partial z}
\end{array}\right)
$$

where $\vec{u}=(u, v, w)$ is the displacement vector at point $M$ and $(x, y, z)$ are the Lagrangian coordinates.

Furthermore, if the transformation is supposed to be homogeneous throughout the thickness of the sample (thinsheet assumption), eq (2) becomes

$$
1=\left(1+\frac{\partial w}{\partial z}\right) \cdot \operatorname{det}\left(\begin{array}{cc}
1+\frac{\partial u}{\partial x} & \frac{\partial u}{\partial y} \\
\frac{\partial v}{\partial x} & 1+\frac{\partial v}{\partial y}
\end{array}\right)
$$

Knowing the spatial derivatives of the in-plane displacement components $(u$ and $v)$ and the initial geometry $(z(x, y))$, the integration of the differential eq (4) gives the out-of-plane component of the displacement $w$ at point $M$.

Once the whole displacement vector field has been calculated, the grid is upgraded, and the displacement field between a next couple of images can be determined; the path of each point on the grid is then calculated step by step. After a first loading increment, the position of each point $M$ no longer corresponds to an integer value of pixel. Naturally, the simplest way to compute the next displacement is to choose the nearest integer pixel location as a basis for the next measurement. By doing so, we add errors to the measurement and deviate from the true Lagrangian measurement. The typical strain increment $\Delta \varepsilon$ between two images is about $510^{-3}$. Consequently, the maximum error on the displacement measurement due to the nearest pixel approximation is around $\Delta \varepsilon / 2$, which corresponds to $2.510^{-3}$ pixels. This small error is reduced by performing a local numerical translation of images $I_{1}$ and $I_{2}$. Using a bilinear interpolation, the correlation zone $C Z$ of each point $M$ is shifted by the decimal value of the previously computed displacement in order to always track the same material zone throughout the test. 
TABLE 1-EXAMPLES OF CPU TIMES PERFORMED ON PENTIUM MMX $166 \mathrm{MHz}$ WITH CZ=20 PIXELS:

(a) FINE-GRID AND LARGE RESEARCH ZONE, (b) COARSE-GRID AND LARGE RESEARCH ZONE AND

(c) FINE-GRID AND SMALL RESEARCH ZONE

\begin{tabular}{cccc} 
& $(\mathrm{a})$ & $(\mathrm{b})$ & $(\mathrm{c})$ \\
& $n=10^{4}$ Points & $n=10^{2}$ Points & $n=10^{4}$ Points \\
& $R Z=103$ Pixels & $R Z=103$ Pixels & $R Z=4$ Pixels \\
\hline CPU Time (s) & 22227 & 222 & 42
\end{tabular}

\section{Differentiation of the Displacement Field}

\section{Local Approximation Technique}

To achieve high-precision measurements of strain and strain rate, the noisy displacement data have to be filtered before computing time and space differentiation. Filtering methods often rely on the spectral decomposition of the signal to be filtered (using fast Fourier transform algorithms) provided the signal is regularly sampled. ${ }^{17,18}$ When localization occurs, the strong evolution of the surface aspect may be responsible for "bad" correlation computation giving incoherent amplitude and orientation of the displacement vector. In our experimental conditions, less than 0.5 percent of the points are concerned by this bad correlation. Because we chose to ignore these points, Fourier filtering methods were eliminated. We implemented a method based on a local least squares approximation of the displacement field to process irregularly sampled data. For the sake of simplicity, this method is illustrated hereafter in the one-dimensional case.

The displacement field is locally fitted within an approximation zone $A Z$. The approximation function $g$ is selected according to the differential operator applied. For space differentiation, we chose a linear approximation function corresponding to a uniform strain along the approximation zone, whereas for time differentiation, we chose a quadratic approximation function corresponding to a locally constant acceleration. Each differential operator is directly applied to its approximation function. Repeating this computation for all points of the grid, the strain fields can be constructed.

\section{Choice of AZ}

The parameter $A Z$ corresponds to a fixed number of nearby points used to compute the local fit. Naturally, its size affects the level of smoothing and, thus, the error on the determined strain. The choice of $A Z$ corresponds to a compromise between smoothing and accuracy: large approximation zones reduce the standard deviation on the strain measurement. So, in the case of a homogeneous strain distribution, large values of $A Z$ can be used to increase the accuracy of the strain computation, whereas in the case of a strain localization, large $A Z$ tends to make the strain field uniform.

\section{Continuity Extension at the Boundary}

A continuity extension of the displacement field is performed at the image boundary in order to keep each point of the grid in the center of its approximation zone. The construction of the extended data is schematically illustrated in Fig. 1. First, the approximation function $g_{1}$ is determined in a neighborhood $A Z_{1}$ of $P_{1}$. Then, the locus of a first extended point $p_{1}^{e x t}$ is chosen on the other side of the boundary; the distance between $p_{1}^{e x t}$ and the boundary is taken equal to the averaged grid step within $A Z_{1}$. The displacement at $p_{1}^{e x t}$ is

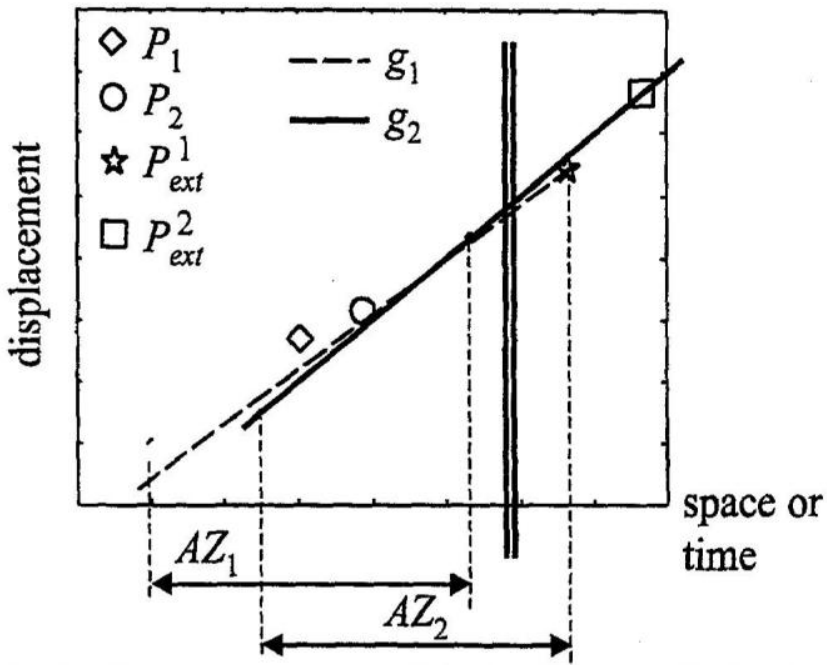

Fig. 1-Continuity extension of the displacement field at the boundary

then calculated using $g_{1}$. This calculation is reiterated using these extended data, and the extension process is stopped once $A Z / 2$ points are created.

\section{Checking the Data Processing}

The validity check of the displacement calculation becomes experimentally very difficult to carry out as soon as the displacement field is no longer that of a rigid body. It is impossible to experimentally impose a given strain field on a real structure. Therefore, the algorithms of correlation and differentiation were tested on several analytic examples based on numerically deformed images. The image $I_{1}$ may be either virtual or real.

\section{Interpolated Real Images}

The initial image is taken under normal shooting conditions. It is interpolated by a bicubic spline and resampled on a deformed grid. The deformation of the grid is related to the image deformation. Naturally, this interpolation is only valid if the variations of light intensity of the original image remain relatively regular.

With this method, it is very difficult to impose or to change the image characteristics (spectral content). To easily modify these characteristics, we developed a digital image creation technique.

\section{Computer-generated Speckle Images}

The virtual images correspond to a distribution of luminous spots on the surface. Each spot is represented by a Gaussian-shaped function. The location $\left(x_{k}, y_{k}\right)$, "size" 


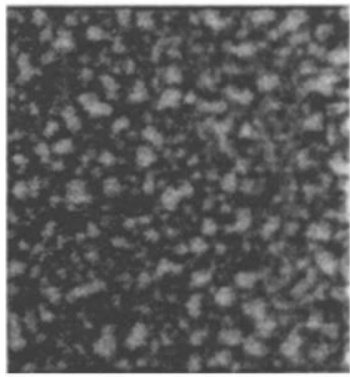

(a)

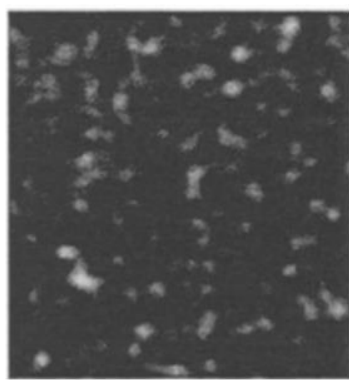

(b)
Fig. 2-(a) Experimental and (b) computer-generated $140 \times$ 140 pixel speckle images

$\left(R_{k}^{x}, R_{k}^{y}\right)$ and intensity $\left(I_{k}^{0}\right)$ of a particular spot (for instance, the $k$ th spot) are randomly chosen. The superposition of these $N_{s}$ spots gives the following analytic definition $I(x, y)$ of the computer-generated speckle image:

$$
I(x, y)=\sum_{k=1}^{N_{s}} I_{k}^{0} e^{-\left(\frac{x-x_{k}}{R_{k}^{k}}\right)^{2}} e^{-\left(\frac{y-y_{k}}{R_{k}^{k}}\right)^{2}} .
$$

By sampling this analytic function on a regularly spaced grid corresponding to the CCD detector, we obtain a computer-generated digital image. Because the analytic definition of this function is known, it can be transformed in various ways: translation, rotation or deformation. Of course, the images are generated so as to obtain histograms and Fourier spectra similar to the real ones. Figure 2 shows an example of real and virtual speckle images.

\section{Checking In-plane Displacement Measurements}

Translation motions applied to computer-generated speckle images show that the mean error on the displacement measurement $\left(\bar{e}_{u}\right)$ depends on the imposed displacement $\left(u_{i m p}\right)$. It is defined by

$$
\vec{e}_{u}=\underset{i}{\text { mean }}\left(u_{\text {comp }}^{i}-u_{i m p}\right),
$$

where $u_{\text {comp }}^{i}$ is the computed displacement at the $i$ th point. The systematic error is approximately sinusoidal, and the period is 1 pixel (see Fig. 3). This result is consistent with previous experimental studies. ${ }^{14,19}$ Henceforth, $\bar{e}_{u}$ will be referred to as the systematic error $\left(\bar{e}_{s y s t}=\bar{e}_{u}\right)$. Thus, in the one-dimensional case, the error on the displacement measurement at the $i$ th point $\left(e_{u}^{i}\right)$ is written as

$$
e_{u}^{i}=e_{s y s t}\left(u_{i m p}\right)+e_{r a n d}^{i},
$$

where $e_{\text {rand }}^{i}$ is the random component of the error at point $i$.

Naturally, we have checked that $e_{\text {rand }}^{i}$ was indeed a random error and that its characteristics (mean value, standard deviation) did not depend on the displacement applied (see Fig. 3). Figure 4 shows the normal distribution of the random error with a zero mean value and a standard deviation of 1.2 $10^{-2}$ pixels. We also observed that $e_{\text {syst }}$ does not depend on the value of $C Z$, whereas $e_{\text {rand }}^{i}$ does: the higher $C Z$, the lower $e_{\text {rand }}^{i}$.

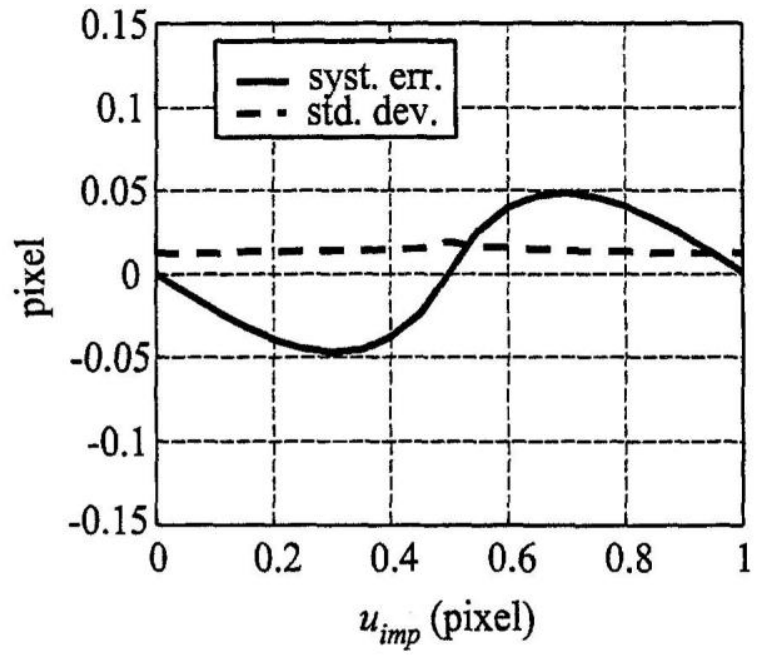

Fig. 3-Evolution of the error on the displacement measurement versus the imposed displacement corresponding to a direct correlation computation $\left(C Z=20\right.$ pixels, $n=10^{2}$ points)

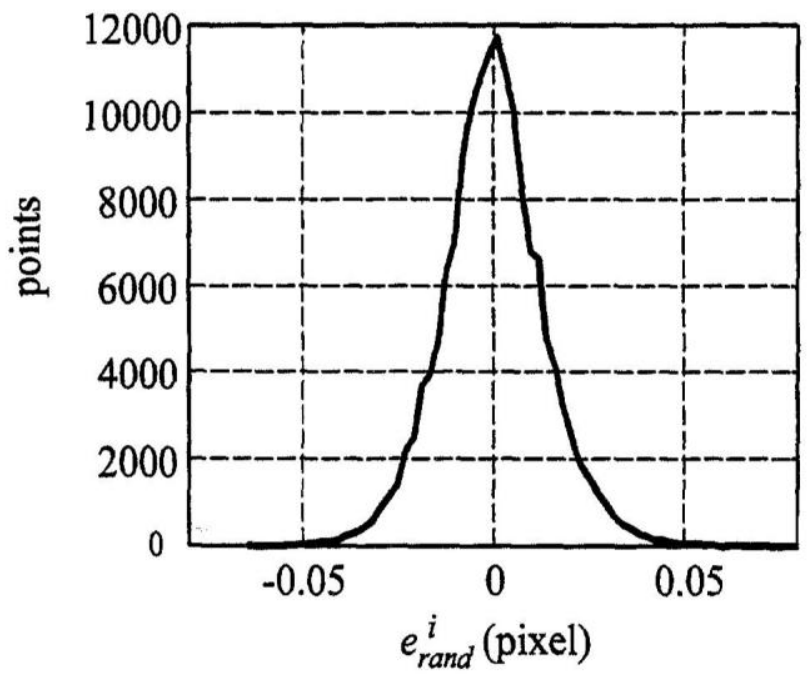

Fig. 4-Density probability function of the random error on the displacement measurements corresponding to a direct correlation computation ( $C Z=20$ pixels, $n=2.10^{5}$ points)

By using different spot sizes, we show that the amplitude of the systematic error depends on the speckle properties (see Fig. 5 and Table 2): the larger the spots, the smaller the systematic error amplitude, whereas $e_{\text {rand }}^{i}$ is not sensibly affected by spot size.

The systematic error is not only due to the chosen way to perform the correlation. We verified that the use of Fourier techniques also leads to similar features. Calculations in the frequency space produce not only a systematic error but also a drift that increases linearly with the imposed displacement (see Fig. 6).

An explanation for the occurrence of the drift is given in Ref. 20. The spectra of two translated images are not exactly the same. On one side of the correlation zone, spots arrive, 


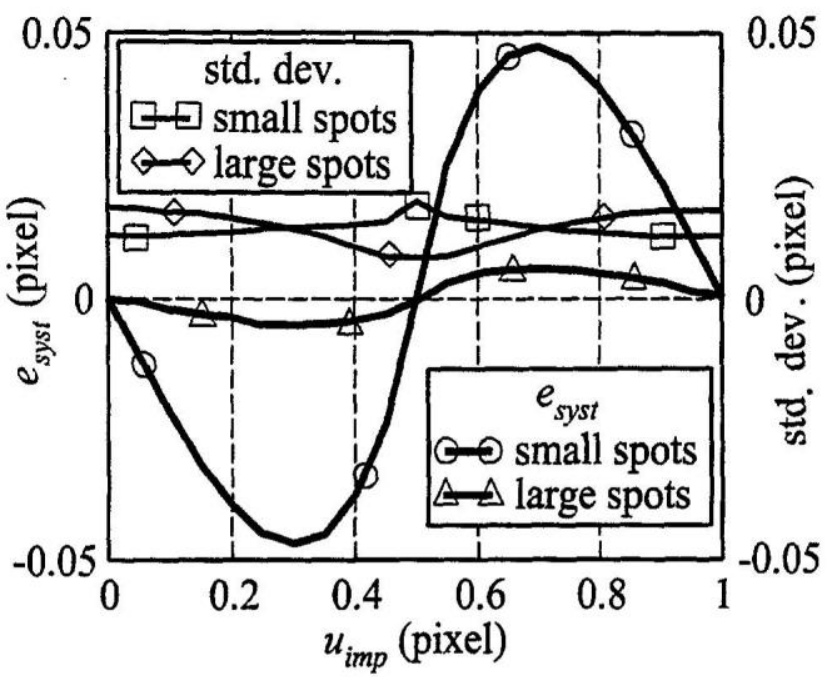

Fig. 5-Systematic error corresponding to a direct correlation computation ( $C Z=20$ pixels, $n=10^{2}$ points). The size of large spots is around $10 \times 10$ pixels, and the size of small spots is $2 \times 2$ pixels

TABLE 2-MAXIMUM SYSTEMATIC ERROR AND STANDARD DEVIATION FOR VARIOUS MEASUREMENT METHODS ON SMALL-SPOT SPECKLE IMAGES: (a) DIRECT CORRELATION COMPUTATION, (b) DIRECT ITERATIVE CORRELATION COMPUTATION, (c) RANDOM-SHIFTING DIRECT CORRELATION COMPUTATION AND (d) FOURIER ITERATIVE CORRELATION COMPUTATION

\begin{tabular}{cccc}
\hline & $\begin{array}{c}\max \left(e_{\text {syst }}\right) \\
\text { (pixel) }\end{array}$ & $\begin{array}{c}\text { Standard } \\
\text { Deviation } \\
\text { (pixel) }\end{array}$ & $\begin{array}{c}\text { CPU Time } \\
\text { (arbitrary } \\
\text { units) }\end{array}$ \\
\hline (a) & $5.10^{-2}$ & $2.10^{-2}$ & 1 \\
(b) & $3.10^{-2}$ & $2.10^{-2}$ & 3 \\
(c) & $10^{-2}$ & $6.10^{-2}$ & 1 \\
(d) & $10^{-3}$ & $6.10^{-3}$ & 20
\end{tabular}

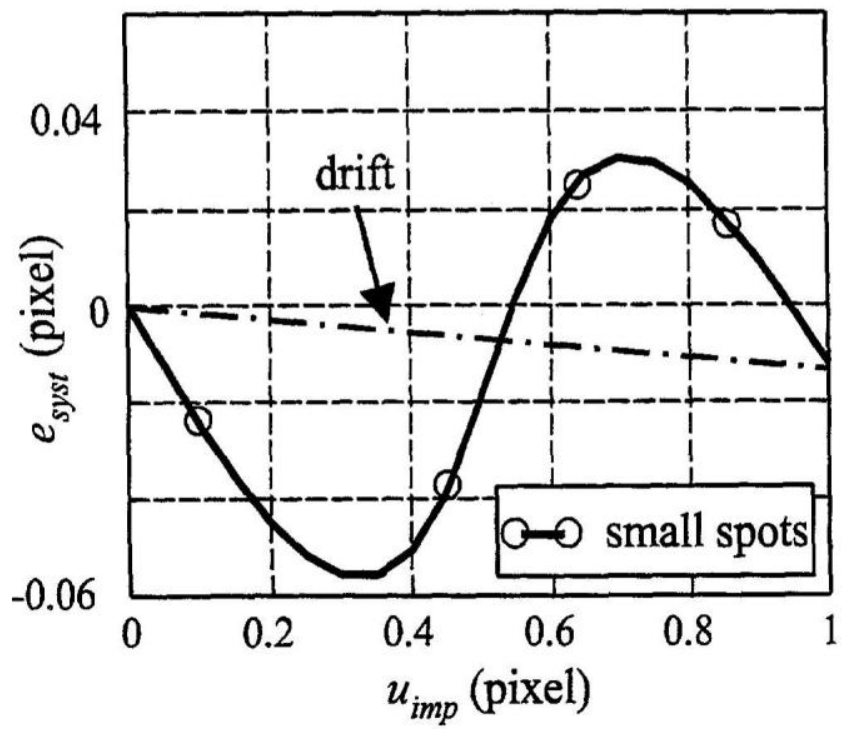

Fig. 6-Systematic error plus drift corresponding to a direct Fourier correlation computation ( $C Z=20$ pixels, $n=10^{2}$ points) whereas on the other side, some of those that were initially present leave the zone. This (small) difference induces an error in the measurement of the displacement that increases with the spots' "flux." A convenient way to remove this drift is to resort to iterative calculations. ${ }^{20}$

Simulations using computer-generated speckle images show that the systematic error stems from the combination of two factors: the numerical integration error deriving from calculating the correlation and the use of the subpixel polynomial interpolation of the intercorrelation function.

By contrast with the random error, the systematic error cannot be easily removed by standard filtering techniques. However, several methods can be used to reduce its influence.

\section{How to Remove $\mathrm{e}_{\text {syst }}$}

With iterative methods, ${ }^{13,20}$ the correlation computation converges gradually toward autocorrelation (zero displacement). As a consequence, these methods suppress, by construction, the systematic error but remain time consuming.

A faster method, developed by in Refs. 14 and 19, is based on an approximation of the systematic error by a sinusoidalshaped function. These works correct the displacement measurement by the value of the corresponding modeled systematic error. However, this method does not take into account the dependence of the systematic error on speckle properties.

Our proposal is to use some characteristics of the systematic error to get rid of this error: 1-pixel periodicity and null average over one period (see Figs. 4 and 5). The aim of this technique is to convert the systematic error into a random one to suppress it by a subsequent filtering. To that end, the correlation zone of each point of the final image is numerically translated by a 1-pixel-amplitude random displacement $\left(u_{\text {rand }}^{i}\right)$ using a bilinear interpolation of the image. The computed displacement of the $i$ th point can thus be written as

$$
u_{\text {comp }}^{i}=\left(u_{\text {imp }}^{i}+u_{\text {rand }}^{i}\right)+e_{\text {syst }}\left(u_{\text {imp }}^{i}+u_{\text {rand }}^{i}\right)+e_{\text {rand }}^{i} .
$$

Because it depends on a random displacement, the systematic error computed at each point will become independent of that computed for its neighbors; it is randomly redistributed throughout the whole image (see Fig. 7). Then, $u_{\text {rand }}^{i}$ is subtracted to $u_{\text {comp }}^{i}$, and, using the property of null average, it is possible to greatly reduce the amplitude of the superimposed random error $e_{s y s t}\left(u_{i m p}^{i}+u_{\text {rand }}^{i}\right)$ using the smoothing method described earlier.

Figure 7 shows the displacement profile along the sample axis between two homogeneously deformed images, obtained using direct correlation $\left(u_{\text {direct }}\right)$ or random shifting $\left(u_{\text {rand.shift }}\right)$ computations. We can observe the influence of the systematic error on the displacement measurements and the efficiency of the random shifting method. Figure 8 gives the strain profiles related to Fig. 7. We can also observe that the maximum error for strain has been considerably reduced.

Table 2 shows the comparative performances of the different measurement techniques that we implemented. In the case of translation motions, the most efficient but most timeconsuming method is the Fourier iterative computation (d). Direct methods $(a, b, c)$ are much faster, and the randomshifting direct correlation computation (c) gives a good compromise between speed and accuracy. 


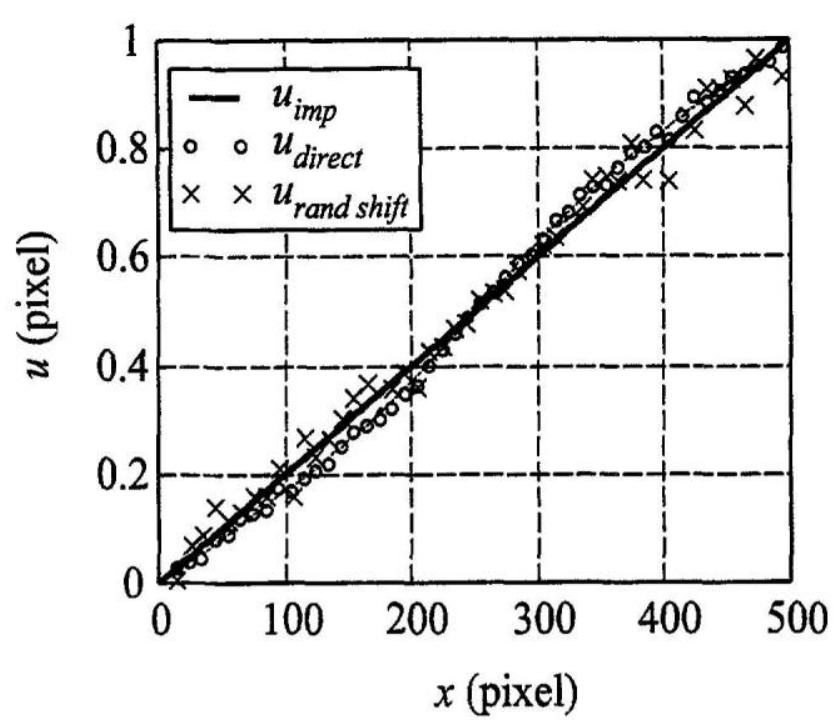

Fig. 7-Displacement profiles using either direct or random shifting methods $(C Z=20$ pixels)

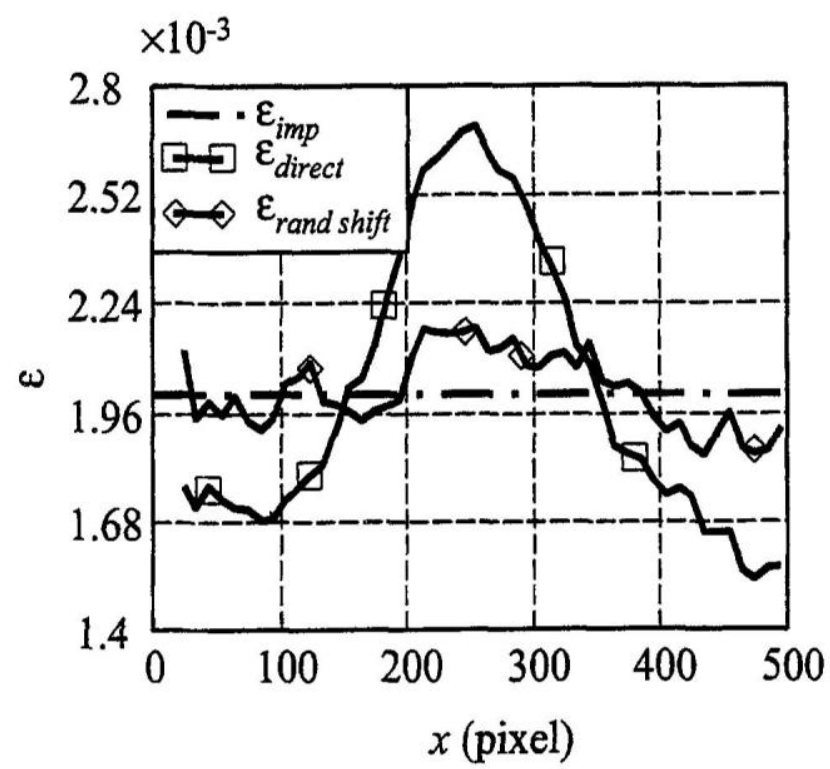

Fig. 8-Strain profiles related to displacement profiles of Fig. $7(A Z=50$ pixels $)$

The order of magnitude of the CPU time consumption is given in arbitrary units. With a Pentium MMX $166 \mathrm{MHz}$, and for $C Z=20$ pixels and $R Z=3$ pixels, this unit achieves 238 subsets per second. Note that direct correlation and randomshifting direct correlation algorithms have similar CPU time consumption. This stems from the shifting operation, which is much faster than the subsequent correlation computation.

\section{Sensitivity to Rotation Motions}

Direct correlation computations can give only the local translation components at any point of the grid. They are not able to determine the local rotation of $C Z$. Nevertheless, this can be estimated, provided the rotation angle between two images remains small. We studied the sensitivity of the correlation computation with regard to rotation movements using numerically rotated images. Imposed incremental rotation angles were taken between 0 deg and $20 \mathrm{deg}$. We noticed that the variations of the intercorrelation function $\bar{\varphi}$ were strongly affected by rotation motions. For instance, Figs. 9(a) and 9(b) show the variations of $\bar{\varphi}$ in the vicinity of $M\left(i_{0}, j_{0}\right)$ using the same $R Z$ in the case of a translation motion and of a 20 deg rotational movement, respectively. We can clearly see that there is only one correlation peak within $R Z$ for the translation, whereas several peaks can coexist in the rotation case. Besides, the peak that corresponds to the rotational movement is not necessarily the highest. To find the right peak, we compute a first estimate of the displacement field using small values of $R Z$.

The choice of a small $R Z$ allows only for the calculation of small displacements, which correspond here to points located in the vicinity of the rotation center. Far from this center, the data processing does not provide correct values of displacements. Once the rotation angle is determined, the same low value of $R Z$ can be used for the whole image, providing a single correlation peak within the research zone. This method gave correct results for incremental rotation less than $20 \mathrm{deg}$.

In Fig. 10, the evolution of the error was plotted for angles varying from $0 \mathrm{deg}$ to $20 \mathrm{deg}$. Note that an angle of $20 \mathrm{deg}$ between two consecutive images can still be found with an approximate mean error of $0.1 \mathrm{deg}$ and a local accuracy of $1 \mathrm{deg}$.

\section{Checking Out-of-plane Displacement Component}

The computational procedure providing the out-of-plane displacement component is checked experimentally by recording images of a steel sample mechanically loaded until localized necking occurs. The sample was then unloaded and removed from the testing machine, and its dimensions were measured using a three-axis measurement machine (Renault Automation, resolution approximately $1 \mu \mathrm{m}$ ). The temporal evolution of the out-of-plane displacement component was computed using eq (4). The elastic strain being neglected compared with the plastic strain, the final computed geometry was then compared to the measured one. Figure 11 shows the comparison between the computed and measured thicknesses of the sample along its longitudinal axis. Results are in good agreement and show the global consistency of the hypotheses made throughout data processing.

\section{Checking Strain Measurements}

As mentioned earlier, we decided to distinguish situations in which the deformation is homogeneous from cases in which it is heterogeneous. The differential operators are estimated with noisy displacement data and can be compared with analytic results. The strain resolution mainly depends on the parameter $A Z$, the number of approximation points within $A Z$, the incremental strain between two images and, for a cumulative computation, the number of strain increments. Because we choose to work with a fixed grid step (typically, $G S=5$ pixels), the number of approximation points is given by the value of the parameter $A Z$.

For images deformed in a homogeneous way, the resolution on the strain measurement increases with the use of higher values of parameter $A Z$ (see Fig. 12). The mean error is the difference between the imposed strain increment and its mean measured values. The standard deviation of the local 

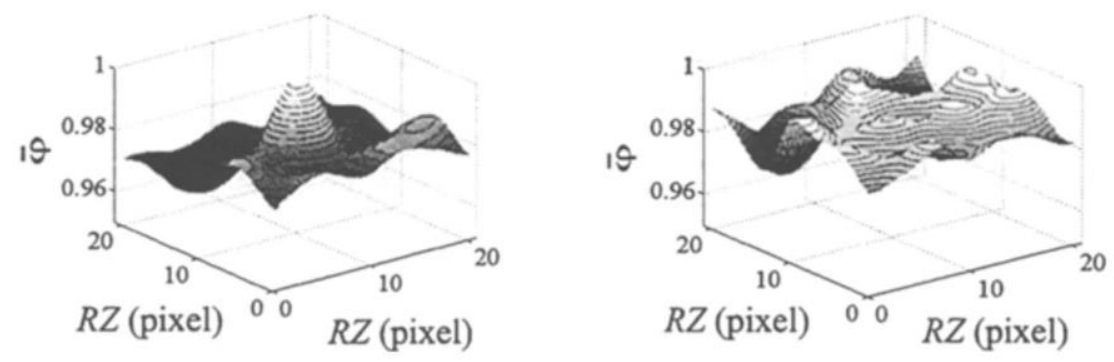

Fig. 9-Intercorrelation function variations: (a) rigid-body translation, (b) rigid-body rotation $(C Z=20$ pixels, $R Z=10$ pixels)

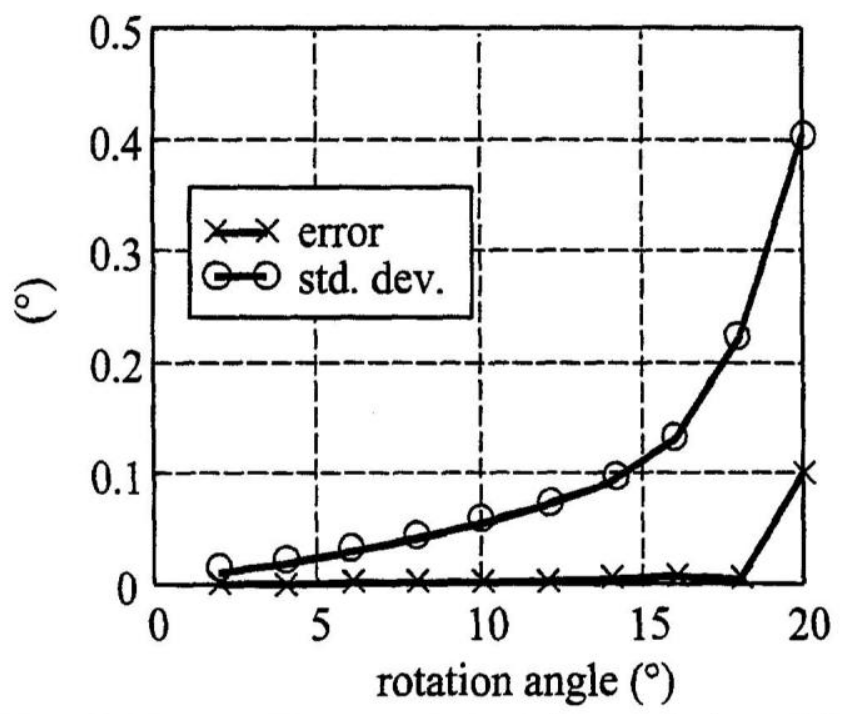

Fig. 10-Error on the computed rotation angle $\left(n=10^{4}\right.$ points)

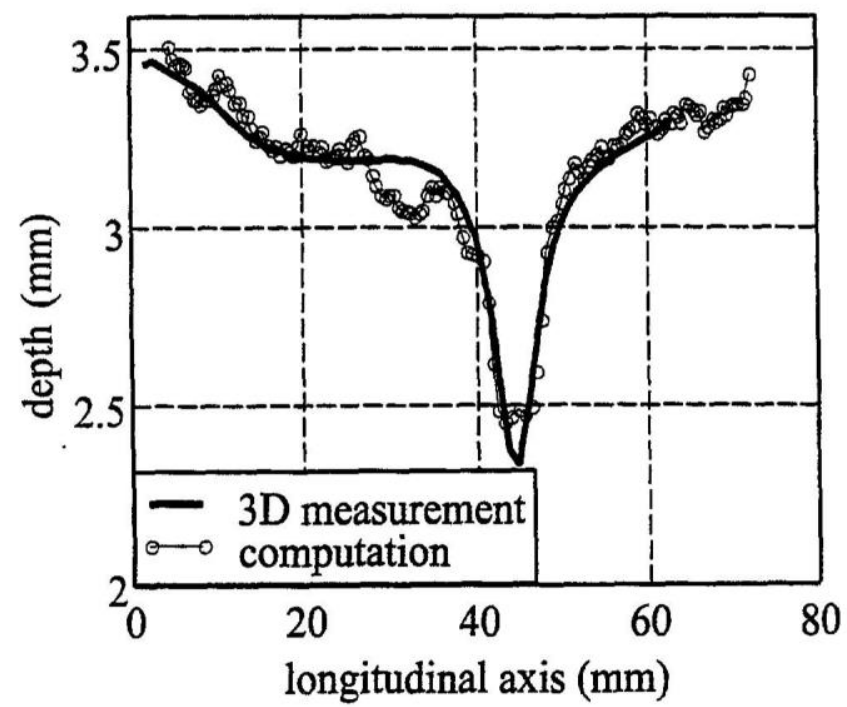

Fig. 11-Comparison between computed and measured outof-plane displacements

strain error is given. Figure 12 also shows that the CPU time consumption varies approximately with the square of $A Z$.

Figure 13 illustrates the dependence of the strain measurement error at fixed $A Z$ according to the incremental strain $(\Delta \varepsilon)$ between the two homogeneously deformed images. The

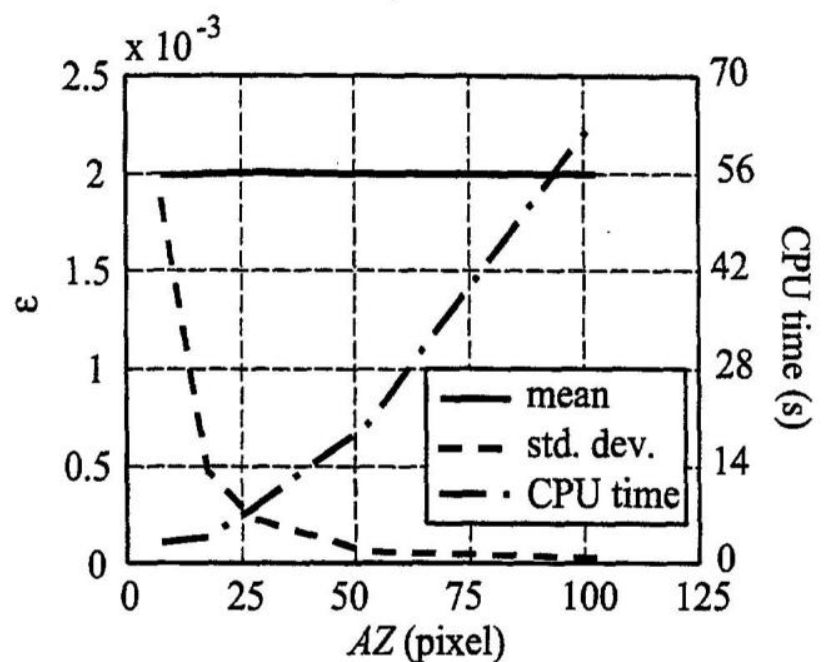

Fig. 12-Strain measurement between homogeneously deformed images ( $n=8500$ points, $C Z=20$ pixels, $\Delta \varepsilon=0.2$ percent)

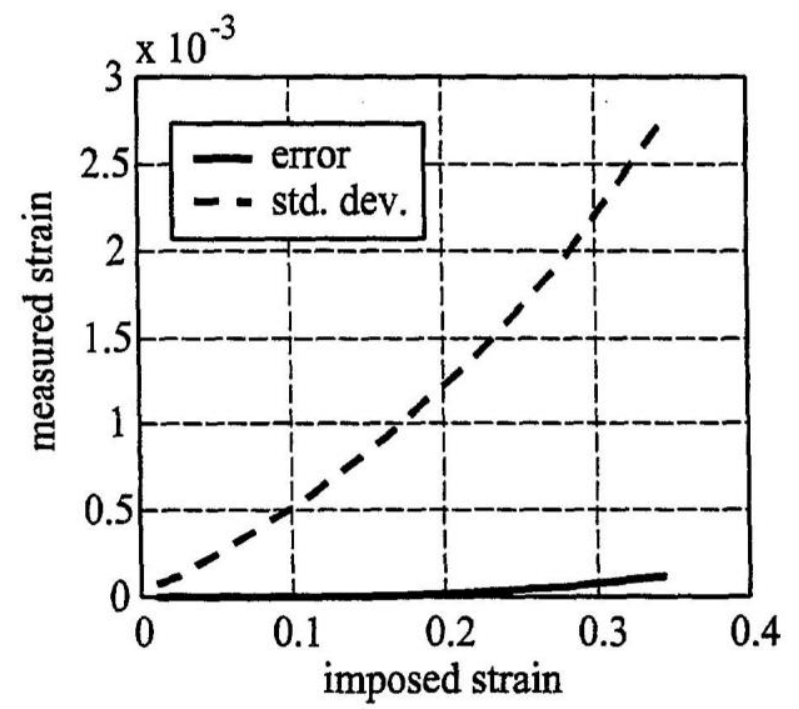

Fig. 13-Evolution of the error on strain measurement according to the incremental strain between the two images ( $C Z=20$ pixels, $A Z=50$ pixels, $n=10^{4}$ points)

mean error remains small (i.e., $2.10^{-4}$ ) even for $\Delta \varepsilon=30$ percent, whereas the amplitude of the local error increases from $2.10^{-4}$ at $\Delta \varepsilon=0.5$ percent to $5.10^{-3}$ at $\Delta \varepsilon=35$ percent.

Figure 14 illustrates a penalizing case corresponding to the existence of a narrow localization zone (size of around 200 


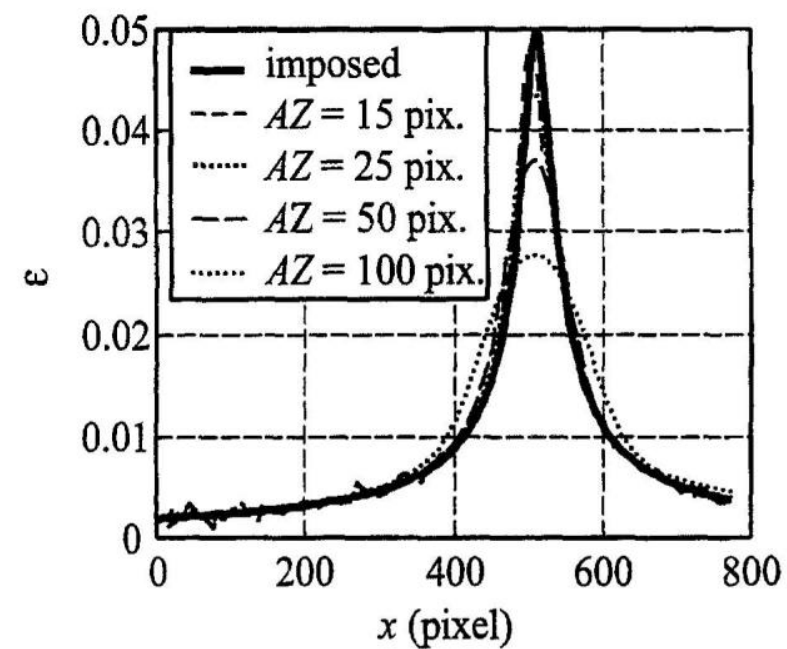

Fig. 14-Strain resolution for the case of a heterogeneous strain field $(C Z=20$ pixels)

pixels). As expected, we verify that increasing $A Z$ tends to enlarge the size of the localization zone and to underestimate the maximum strain, which leads to an increase of the strain measurement error.

Using this kind of example, we found that $A Z$ values ranging from 25 to 50 pixels are a good compromise between precision and CPU time, whether or not the strain field is homogeneous.

The accuracy of the strain measurements is also related to the number of displacement increments. This dependence is illustrated in Fig. 15. The strain is computed by cumulating the incremental displacement vectors while the strain increment between two consecutive images is equal to 0.5 percent. We can see that the mean error remains small $\left(<2.10^{-4}\right)$ whichever the number of increment. We also observe that the local error decreases at first, then increases due to the accumulation of incremental errors. However, the standard deviation reached for an imposed deformation of 35 percent is lower for the case of an incremental calculation compared with the case of a direct one.

\section{Experimental Arrangement}

The experimental setup uses a $100 \mathrm{kN}$ tensioncompression, servomechanic testing machine. The eight-bit digital images are recorded during the test by a CCD camera positioned in front of the sample. The lens axis of the camera is kept fixed with reference to the frame of the testing machine and remains perpendicular to the surface of the sample. We used a Kodak MegaPlus high-resolution camera $1317 \times 1035,6.8 \times 6.8 \mu \mathrm{m}$ square pixels and a Nikon lens (Micro-Nikkor, $f_{0}=55 \mathrm{~mm}$ ). The distance between the camera and the sample is around $1 \mathrm{~m}$.

Concerning the lighting mode, we prefer white light to coherent light because its use is less sensitive to out-of-plane movements and to the increase of the surface roughness due to strain hardening that both disturb the data analysis. Of course, for natural lighting, the sample surface must be contrasted enough to allow correlation computations. In this work, the steel samples are artificially speckled using white paint. The appearance of the sample aspect is illustrated in Fig. 2(a).

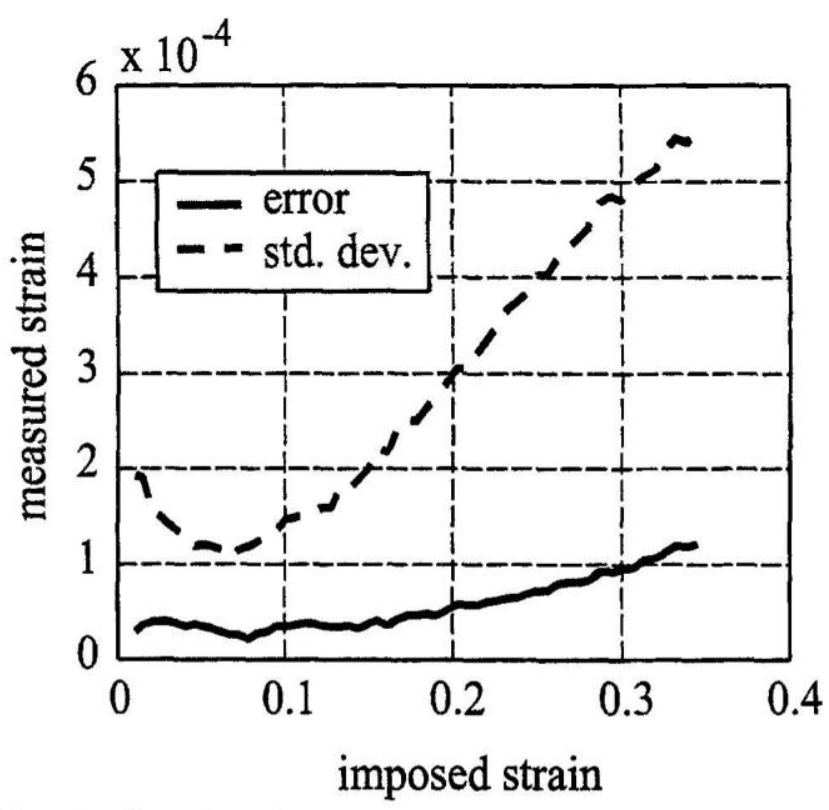

Fig. 15-Evolution of the strain measurement error according to the number of strain increments $(C Z=20$ pixels, $A Z=50$ pixels, $n=10^{4}$ points, $\Delta \varepsilon=0.5$ percent)

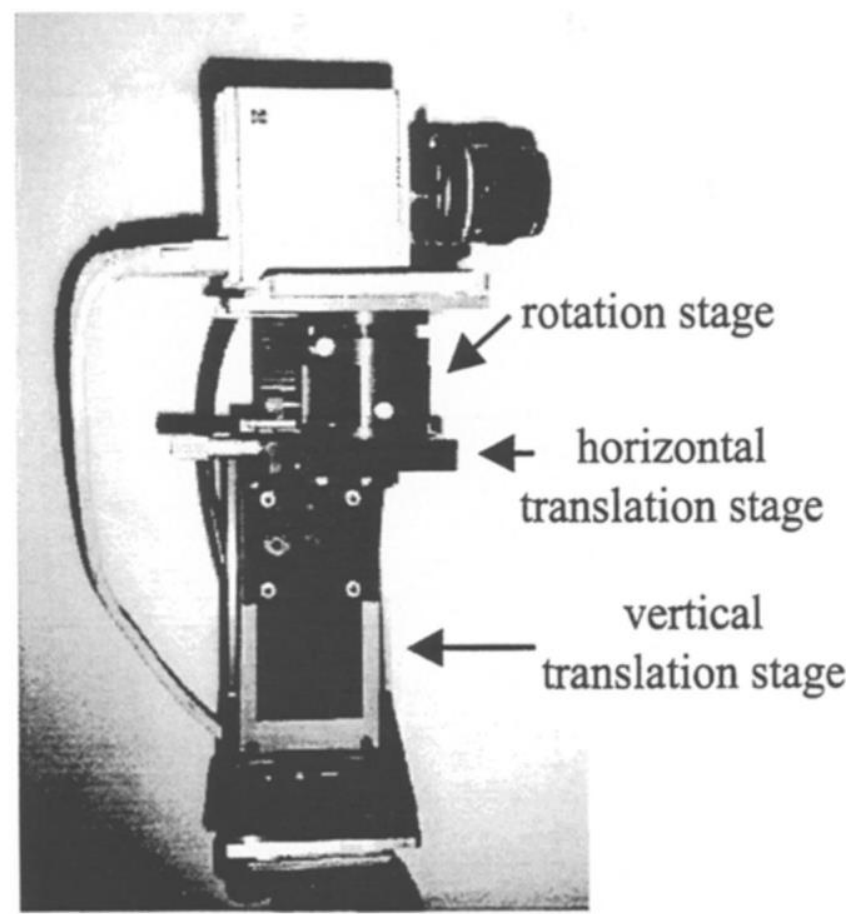

Fig. 16-Camera setting

The camera must be set carefully so that the CCD detector remains parallel to the sample surface, since each outof-plane movement (translation or rotation) distorts the images. In order not to disturb the interpretation of the measurements, it is of great importance to be able to minimize these distortions.

We adopt the following procedure for setting up the camera. After an initial coarse setup-using a three-axis rotation stage (see Fig. 16) for easy tilt corrections-fine adjustment is achieved by vertical translation of the sample in front of the camera using the testing machine. The comparison between 


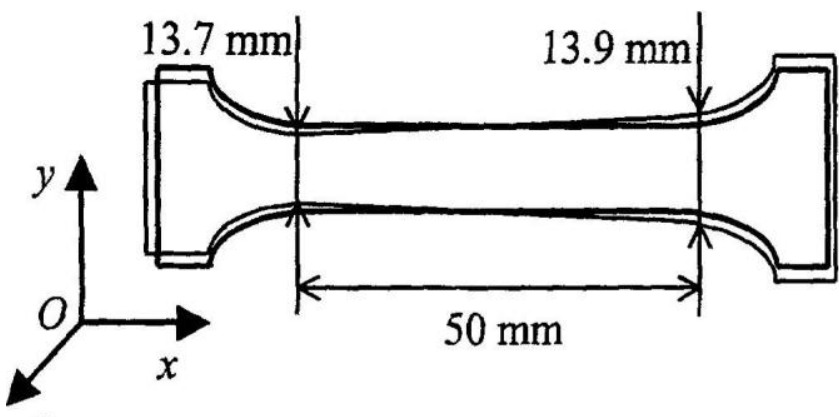

$Z$

Fig. 17-Heuristic sketch of the geometrical sample defects

the strain field associated with this rigid-body motion (identically equal to zero) and the measured strain field enables us to check and optimize the alignment procedure. The typical error on the strain measurements, due to the setting up, is about $1.10^{-4}$ or $2 \cdot 10^{-4}$. Note that this error corresponds approximately to the strain measurement resolution.

\section{Examples of Potential Application}

The experimental setup is designed to study localization phenomena in mild steels. To illustrate the capabilities and the interest of such an approach, we give an outline of results obtained during monotone tensile tests performed at constant cross-head velocity $\left(\nu_{C H}=0.04 \mathrm{~mm} . \mathrm{s}^{-1}\right)$. As long as the strain field remains homogeneous within the gage part of the sample, this kind of loading can also be interpreted in terms of a strain-controlled test $\left(\dot{\varepsilon}_{\text {macro }} \approx 7.10^{-4} \mathrm{~s}^{-1}\right)$. The images corresponding to the sample gage part contain $250 \times 900$ pixels (scale factor $\approx 85 \mu \mathrm{m}$.pixel ${ }^{-1}$ ), and the frame rate is about 0.2 image. $\mathrm{s}^{-1}$.

It is well known that initial geometrical defects in the sample can affect the inception of localization phenomena. ${ }^{9}$ So we tested samples with and without geometrical defects to evaluate the sensitivity of our experimental procedure. These geometrical defects of machining were deliberately created and correspond to variations of the width along the sample axis (trapezoid shape). The amplitude of the geometrical defect is estimated using, once more, the three-axis measurement machine (see Fig. 17).

The amplitude of the geometrical defect being small $(0.2 \mathrm{~mm})$, the macroscopic tensile curves of the two specimens are very similar (see Fig. 18). As expected, the strain reached before fracture of the sample containing an initial defect is lower than that of the defect-free sample. Two particular points named $A$ and $B$ are spotted on the tensile curves. At the end of this section, they will help us to show that although the macroscopic mechanical responses are initially similar, the local strain and strain rate distributions are very different.

Figure 19 shows the three in-plane components of the Hencky strain tensor $\left(\varepsilon^{H}\right)$ for the sample containing an initial geometrical defect when the applied macroscopic strain $\varepsilon_{\text {macro }}$ is 12 percent (point A, Fig. 18). We can also remark that the strain state within the sample is coherent with a simple tension state: shear strain concentrations are observed in the grip sections of the specimen and are nonmeasurable in the gage part, whereas the lateral contraction ratio $\bar{v}=-\varepsilon_{x x}^{H} / \varepsilon_{y y}^{H}$ is about 0.5. Apart from the grip sections, the strain field

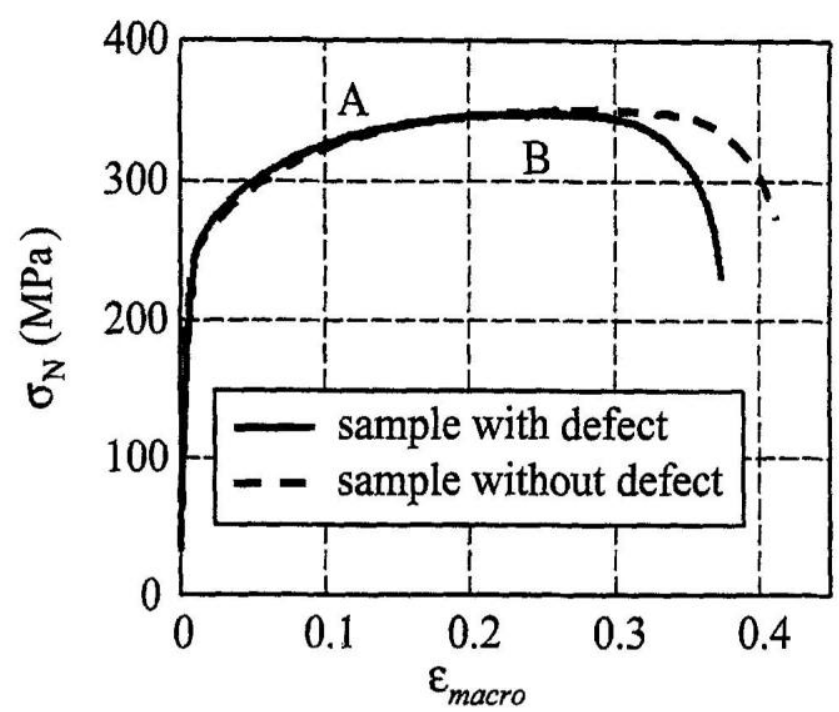

Fig. 18-Macroscopic tensile curves: nominal stress $\sigma_{N}=$ $F / S_{0}$ versus conventional strain $\varepsilon_{\text {macro }}=\Delta l / l_{0}$, where $F$ is the applied load, $S_{0}$ is the initial cross section and $\Delta l$ and $l_{0}$ are the elongation and the initial gage length, respectively

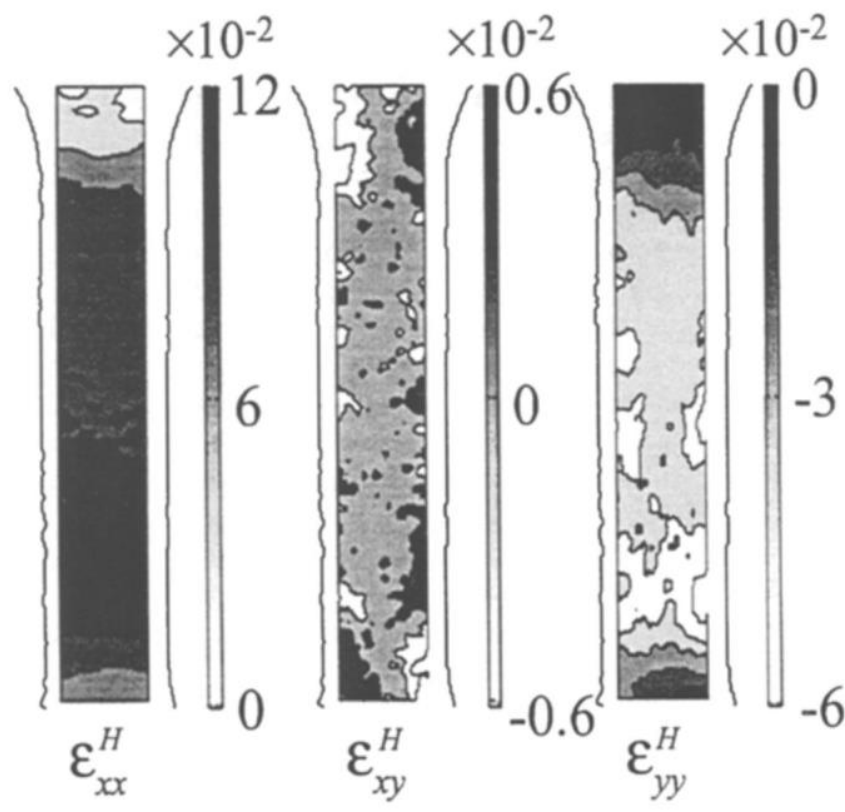

Fig. 19-Spatial distributions of the three in-plane components of the Hencky strain tensor $\varepsilon^{H}$, measured on the sample containing an initial geometrical defect, when the applied macroscopic strain $\varepsilon_{\text {macro }}$ is 12 percent

is heterogeneous within the gage part of the sample; $\varepsilon_{x x}^{H}$ is higher in the lower end of the sample. Naturally, the strain heterogeneity is concentrated in the neighborhood of the geometrical defect where the initial cross section of the sample is the smallest.

Because the local strain state corresponds to simple tension, and because the strain distribution is fairly homogeneous along the sample width (i.e., $O y$-axis), it is possible to summarize all the available two-dimensional information for a given loading state into a one-dimensional representation. For that purpose, we plot only the axial distribution of the tension component of the strain tensor. Figure 20 represents the tension component of the Hencky strain tensor $\left(\varepsilon_{x x}^{H}\right)$ of 


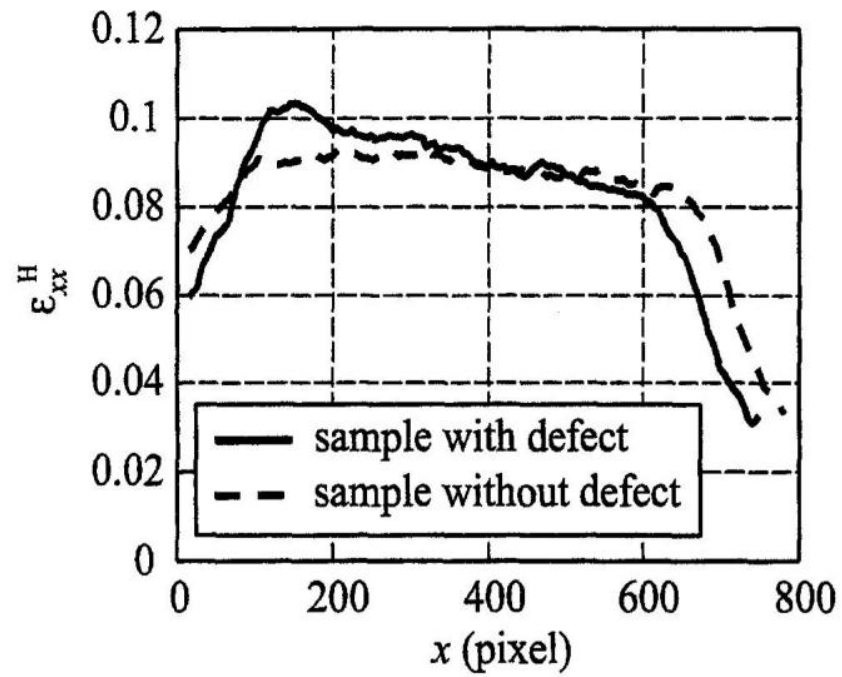

Fig. 20-Longitudinal profile $\varepsilon_{x x}^{H}$ when the applied macroscopic strain $\varepsilon_{\text {macro }}$ is 12 percent

the points located along the sample longitudinal axis (i.e., $O x$-axis). With this representation, we can then compare the amplitude of strain heterogeneity of the two samples.

Strain-rate measurements will provide information about the onset and development of the strain localization. We will adopt the same one-dimensional description of the strain rate state by plotting the tension component of the Lagrangian strain rate tensor $\left(\dot{E}_{x x}\right)$ of the points located along the sample longitudinal axis. To observe, in the same graph, the time evolution of the strain rate distribution, the strain rate profiles are piled in chronological order. In addition, contour plots are drawn and the strain rate intensity is colored in gray scale to further simplify the data interpretation. The abscissa axis stands for time, and the ordinate axis corresponds to space (see Figs. 21 and 22). The loading curve is superimposed on the graph to link global and local aspects of the mechanical response. The horizontal axis (initially time) now stands for the macroscopic strain, and the vertical axis stand for the normalized load.

Figure 21 shows the time evolution of the strain rate for the sample containing a geometrical defect. The strain rate field gradually concentrates along the sample axis, first giving rise to a diffuse necking, then to a localized necking. It is important to note that this concentration takes place early on during the loading in the sense that a strain rate heterogeneity appears during the strain hardening long before the maximum load is reached. Remember that according to some theories, this maximum, which corresponds to the so-called Considère strain $\left(\varepsilon_{\text {macro }}(B)=26\right.$ percent), is associated with the apparition of diffuse necking. At point $B$, we observe that the localization, in terms of strain rate, already covers 30 percent of the gage zone.

During the sample softening, the effects of localization strongly intensify and, finally, lead to the sample fracture.

Figure 22 shows the response of a defect-free sample to the same loading. The onset of the strain rate localization emerges later on during the loading. A slight heterogeneity can be observed on the lower end of the sample: point $A\left(\varepsilon_{\text {macro }}(A)=12\right.$ percent). This strain is less than half of the Considère strain amplitude, for which $\varepsilon_{\text {macro }}(B)=$ 27 percent.

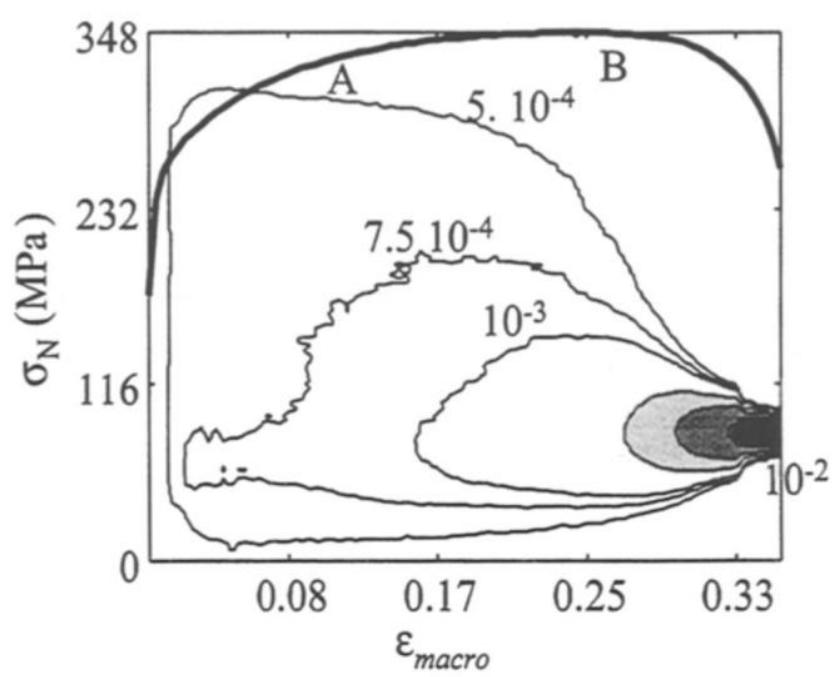

Fig. 21-Time evolution of the longitudinal strain rate $\dot{E}_{x x}(x, 0, t)$ in $\mathrm{s}^{-1}$ for a monotone tensile test performed at constant strain rate $\left(\dot{\varepsilon}_{\text {macro }} \approx 7.10^{-4} \mathrm{~s}^{-1}\right)$ on a sample of steel containing an initial geometrical defect

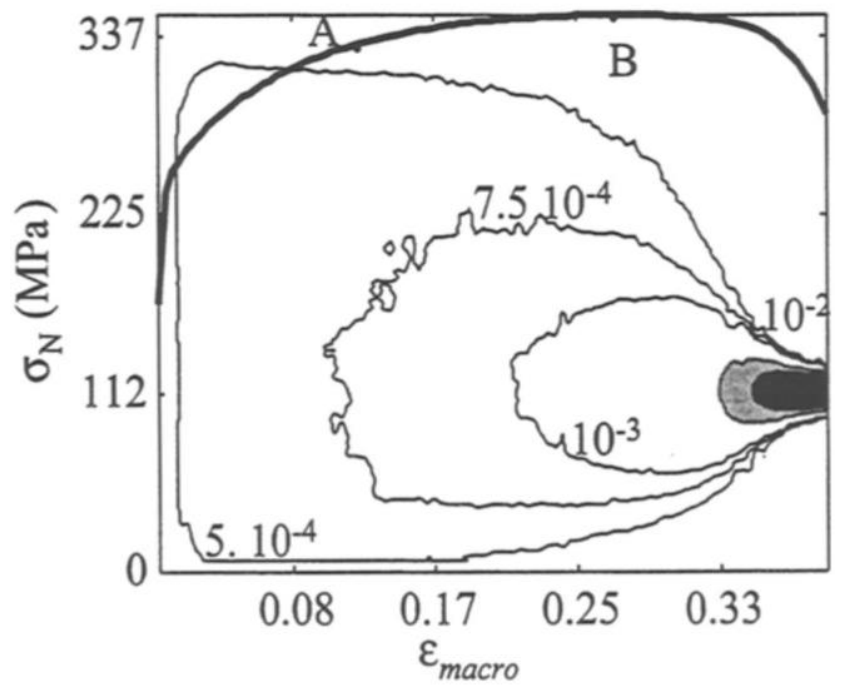

Fig. 22-Time evolution of the longitudinal strain rate $\dot{E}_{x x}(x, 0, t)$ in $\mathrm{s}^{-1}$ for a monotone tensile test performed at constant strain rate $\left(\dot{\varepsilon}_{\text {macro }} \approx 7.10^{-4} \mathrm{~s}^{-1}\right)$ on a sample of steel without any detectable initial geometrical defect

In all the tests performed, the necking is progressive. The presence of a defect in the initial geometry of the sample plays the role of a catalyst and simply brings forward the inception of the localization.

\section{Concluding Comments}

This experimental approach provides the time evolution of various in-plane kinematic variables (displacement, velocity, acceleration, strain, strain rate, rate of kinetic energy and so on). Assuming plastic incompressibility allows the computation of the out-of-plane components. The validity check of the data processing shows that correlation techniques provide results sufficiently accurate to study the inception and the evolution of the localization phenomena. Furthermore, 
we show that direct correlation computations are subject to a systematic error, which can be efficiently removed using a technique of random shifting.

Applied to mild steels, the experimental approach provides results suggesting an early and progressive development of the diffuse and localized necking. The experiments also indicate that the early inception of the diffuse necking is not solely the consequence of geometrical defects. An immediate implication of this precocity is that the gage part of the specimen must be considered as a structure and no longer as a uniformly strained and stressed elementary volume. This fact is currently difficult to reconcile with theoretical views, since standard formalisms are based on the subtle knowledge of the constitutive equations. ${ }^{3-6,8,9}$ As recalled in the first section, these phenomenological equations are identified on the basis of tests, such as the standard tensile test, for which the existence of homogeneous strain and stress fields is very often implicitly assumed. The inception of strain localization leads to the heterogeneity of the stress field, which no longer corresponds to that of the so-called simple tensile test. The knowledge of the load applied to the sample is no longer sufficient to determine the stresses developed within the sample. Constructing the normal stress-strain curve may become hazardous and makes the identification of one-dimensional constitutive equations more complicated.

\section{References}

1. Ladevèze, P., Mécanique non linéaire des structures: Nouvelles approches et méthodes de calcul non incrémentales, Hermès, Paris (1996).

2. Considere, A., "Mémoire sur l'emploi du fer et de l'acier dans les constructions," Annales des Ponts et Chaussées, 9, 574 (1885).

3. Hill, R., "A General Theory of Uniqueness and Stability in Elasticplastic Solids," J. Mech. Phys. Solids, 6, 236-249 (1958).

4. Rice, J.R., "The Localization of Plastic Deformation," Theory of Applied Mechanics, W.T. Koiter, ed., North-Holland, Amsterdam (1976).
5. Bai, Y.L., "Thermo-plastic Instability in Simple Shear," J. Mech. Phys. Solids, 30, 195-207 (1982).

6. Fressengeas, C. and Molinari, A., "Inertia and Thermal Effects on the Localization of Plastic Flow," Acta Metall., 33, 387-396 (1985).

7. Marchand, A. and Duffy, J., "An Experimental Study of the Formation Process of Adiabatic Shear Bands in a Structural Steel," J. Mech. Phys. Solids, 36, 251-283 (1988).

8. Swift, H.W., "Plastic Instability under Plane Stress," J. Mech. Phys. Solids, 1, 1-18 (1952).

9. Marciniak, Z and Kuczynski, K., "Limit Strains in the Process of Stretch Forming Sheet Metal," Int. J. Mech. Sci., 9, 609-620 (1967).

10. Zener, C. and Hollomon, J.H., "Effect of Strain-rate upon Plastic Flow of Steel," J. Appl. Phys., 15, 22-32 (1944).

11. Bailey, H.H., Blackwell, F.W., Lowery, C.L., and Ratkovic, J.A., "Image Correlation: Part I. Simulation and Analysis," Report prepared for United States Air Force Project RAND, R-2057/l-PR (1976).

12. Chu, T.C., Ranson, W.F., Sutton, M.A., and Peters, W.H., "Application of Digital-image-correlation Techniques to Experimental Mechanics," EXPERIMENTAL MECHANICS, 25, 232-244 (1985).

13. Bruck, H.A., McNeill, S.R., Sutton, M.A., and Peters, W.H., "Digital Image Correlation Using Newton-Raphson Method of Partial Differential Correction," EXPERIMENTAL MECHANICS, 29, 261-267 (1989).

14. Choi, S. and Shah, S.P., "Measurement of Deformations on Concrete Subjected to Compression Using Image Correlation," EXPERIMENTAL MECHANICS, 37, 307-313 (1997).

15. Berthaud, Y., Calloch, S., Collin, F., Hild, F., and Ricotti, Y., "Analysis of the Degradation Mechanisms in Composite Materials through a Correlation Technique in White Light," IUTAM Symposium on Advanced Optical Methods and Application in Solid Mechanics, Poitiers University, France (1998).

16. Oulamara, A., Tribillon, G., and Duvernoy, J., "Subpixel Speckle Displacement Measurement Using a Digital Processing Technique," J. Mod. Opt., 37, 1201-1211 (1988).

17. Brigham, E.O., The Fast Fourier Transform, Prentice Hall, Englewood Cliffs, $N J$ (1974).

18. Bellanger, $M$., Traitement numérique du signal-Théorie et pratique, Masson, Paris (1995).

19. lenny, P. and Forner, A., "Extensométrie bidimensionnelle: Calibration et incertitudes de mesure," Conférence Mesures sans contact par procédés optiques, Palavas-les-Flots, France (1995).

20. Thesing, I., "Mesure par corrélation des déplacements et des rotations à partir de figures granulaires," Rapport, LMT Cachan, France (1996). 